\title{
A FRAMEWORK FOR FRACTIONAL HARDY INEQUALITIES
}

\author{
Bartłomiej Dyda and Antti V. Vähäkangas
}

University of Bielefeld, Faculty of Mathematics

Postfach 1001 31, D-33501 Bielefeld, Germany; dyda@math.uni-bielefeld.de, and Wrocław University of Technology, Institute of Mathematics and Computer Science Wybrzeże Wyspiańskiego 27, 50-370 Wrocław, Poland; bdyda@pwr.wroc.pl

University of Helsinki, Department of Mathematics and Statistics P.O. Box 68, FI-00014 University of Helsinki, Finland; antti.vahakangas@helsinki.fi

\begin{abstract}
We provide a general framework for fractional Hardy inequalities. Our framework covers, for instance, fractional inequalities related to the Dirichlet forms of some Lévy processes, and weighted fractional inequalities on irregular open sets.
\end{abstract}

\section{Introduction}

The objective of the present paper is to study inequalities of the general form

$$
\int_{D} \frac{|u(x)|^{p}}{\phi\left(\delta_{x}\right)} \mu(d x) \leq c \int_{D} \int_{D \cap B\left(x, R \delta_{x}\right)} \frac{|u(x)-u(y)|^{p}}{\phi\left(\delta_{x}\right) \delta_{x}^{d}} \mu(d y) \mu(d x) \quad c, R>0,
$$

on metric measure spaces $(X, \rho, \mu)$, with the emphasis on $X=\mathbf{R}^{d}$ equipped with the Euclidean distance and the Lebesgue measure. We write $\delta_{x}=\operatorname{dist}(x, X \backslash D)$ and $D \subset X$ is a possibly irregular open set. The function $\phi:(0, \infty) \rightarrow(0, \infty)$ is a 'perturbation' of a power function $t \mapsto t^{\eta}$ for some $\eta \in \mathbf{R}$, and the exponent $p$ satisfies $0<p<\infty$.

Our main result, Theorem 5 in $\S 3$, brings together two so-far distinct lines along which the fractional Hardy inequality has been generalised: one of them related to the function $\phi$, and the other to the regularity of the open set $D \subset X$. Let us present the Euclidean version of our main result here; Theorem 1 below is a combination of Theorem 5 and Propositions 6 and 10.

Theorem 1. Let $0<p<\infty, H \in(0,1]$ and $\eta \in \mathbf{R}$. Suppose that $D \neq \emptyset$ is a proper $\kappa$-plump open set in $\mathbf{R}^{d}$ and $\phi:(0, \infty) \rightarrow(0, \infty)$ is a function so that either condition $(T)$ or condition $(F)$ holds:

(T) $\overline{\operatorname{dim}}_{A}(\partial D)<d-\eta, D$ is unbounded, and $\phi \in \operatorname{WUSC}\left(\eta, 0, H^{-1}\right)$;

(F) $\underline{\operatorname{dim}}_{A}(\partial D)>d-\eta, D$ is bounded or $\partial D$ is unbounded, and $\phi \in \operatorname{WLSC}(\eta, 0, H)$. Then inequality (1.1) holds for all measurable functions $u$ for which the left hand side is finite.

For the definitions of plumpness and Assouad dimensions $\overline{\operatorname{dim}}_{A}$ and $\underline{\operatorname{dim}}_{A}$, we refer to Section 2; the classes WUSC and WLSC are defined in Definition 3.1. Let

doi:10.5186/aasfm.2014.3943

2010 Mathematics Subject Classification: Primary 26D15, 46E35, 31C25, 26A12.

Key words: Fractional Hardy inequality, fractional Laplacian, regularly varying function, Assouad dimension, uniform fatness, irregular domain. 
us remark that inequality (1.1) fails for nonzero constant functions. This means that under the assumptions of Theorem 1 , the integral $\int_{D} \phi\left(\delta_{x}\right)^{-1} d x$ must be divergent, on the other hand, it shows that the assumption of the left hand side of (1.1) being finite is essential. The relatively simple proof of our main result is a refinement of the techniques in [7] where, e.g., Hardy inequalities (1.3) with $\beta=0$ in case of bounded Lipschitz domains are established.

Theorems 1 and 5 provide a general framework for fractional Hardy inequalities, as they allow for both general open sets $D$ and functions $\phi$ simultaneously. In the sequel, we will state separate corollaries in each of these directions to make the exposition of our framework transparent. First, instead of considering classes WUSC and WLSC, we will confine ourselves to the well-known regularly varying functions. Let us remind that $\phi$ is called regularly varying at origin (resp. infinity) with index $\eta$, if

$$
\frac{\phi(\lambda x)}{\phi(x)} \rightarrow \lambda^{\eta}
$$

when $x \rightarrow 0_{+}$(resp. when $x \rightarrow \infty$ ) for every $\lambda>0$. In the following corollary the geometry of the underlying domain $\mathbf{R}^{d} \backslash\{0\}$ is particularly simple.

Corollary 2. Suppose that $\phi:(0, \infty) \rightarrow(0, \infty)$ is a regularly varying function at origin of index $\rho_{0}$, a regularly varying function at infinity of index $\rho_{\infty}$, and is bounded and bounded away from zero on every compact subset of $(0, \infty)$. Suppose that either $0<\rho_{0}, \rho_{\infty}<d$ or $\rho_{0}, \rho_{\infty}>d$. Let $0<p<\infty$. Then there exists a constant $c=c(\phi, d, p)$ such that inequality

$$
\int_{\mathbf{R}^{d} \backslash\{0\}} \frac{|u(x)|^{p}}{\phi(|x|)} d x \leq c \int_{\mathbf{R}^{d} \backslash\{0\}} \int_{\mathbf{R}^{d} \backslash\{0\}} \frac{|u(x)-u(y)|^{p}}{\phi(|x-y|)|x-y|^{d}} d y d x
$$

holds for every measurable function $u$ for which the left hand side is finite.

A proof of this corollary can be found in $§ 3.4$. Inequalities like (1.2) have been studied in [15] for weights of more general (but also more complicated) form and $p>1$, and in $[14,15,22]$ in the one-dimensional case. The forms appearing on the right hand side of (1.2) for $p=2$ (and for more general domains) are, at least for some functions $\phi$, the Dirichlet forms of certain Lévy processes, which are being extensively studied, see e.g. [13, 20,33] and [5, Section 4.1].

To discuss our results for irregular open sets, we confine ourselves to weighted fractional Hardy inequalities in $\mathbf{R}^{d}$, i.e., we consider the function $\phi(t)=t^{s p-\beta}$ with $d+s p \geq 0$, in which case inequality (1.1) yields

$$
\int_{D} \frac{|u(x)|^{p}}{\delta_{x}^{s p}} \delta_{x}^{\beta} d x \leq c \int_{D} \int_{D} \frac{|u(x)-y(y)|^{p}}{|x-y|^{d+s p}} \delta_{x}^{\beta} d y d x .
$$

An open set $D \subset \mathbf{R}^{d}$ is said to admit $(s, p, \beta)$-Hardy inequality, if inequality (1.3) holds for all functions $u \in C_{0}^{\infty}(D)$ (i.e., smooth with compact support in $D$ ) with $c$ independent of $u$. There has been recent interest in $(s, p, 0)$-Hardy inequalities in connection with the boundary regularity of an open set $D$, we refer to $[10,16,17,18]$. In another direction, the sharp constants for fractional Hardy-type inequalities on general domains are obtained in [27], where the distance is replaced with an averaged pseudo distance. In [9] these results are further refined; let us also mention the other related papers $[4,8,11,12,34]$. 
The non-fractional counterpart of inequality (1.3) has also been studied. Namely, the following weighted $(p, \beta)$-Hardy inequality, with $c>0$,

$$
\int_{D} \frac{|u(x)|^{p}}{\delta_{x}^{p}} \delta_{x}^{\beta} d x \leq c \int_{D}|\nabla u(x)|^{p} \delta_{x}^{\beta} d x
$$

holds for every $u \in C_{0}^{\infty}(D)$ if $D$ is a bounded Lipschitz domain, $1<p<\infty$, and $\beta<p-1$, [32]. More generally, an open set admits a $(p, \beta)$-Hardy inequality if the complement $D^{c}=\mathbf{R}^{d} \backslash D$ is either sufficiently 'thin' or 'fat'. For instance, an open set $D$ admits a $(p, 0)$-Hardy inequality if $D^{c}$ is $(1, p)$-uniformly fat and $1<p<\infty$, [26]. The $(1, p)$-fatness of $D^{c}$ is also known to be sufficient for certain $(p, \beta)$-Hardy inequalities, [24, 37]. A deeper understanding of the 'thin' vs. 'fat' dichotomy is reached in an independent recent study [23], where an open set $D \subset X$ is shown to admit a $(p, \beta)$-Hardy inequality if $D^{c}=X \backslash D$ sufficiently thin or fat, measured in terms of upper and lower Assouad dimension $\left(\overline{\operatorname{dim}}_{A}\right.$ and $\left.\underline{\operatorname{dim}}_{A}\right)$, respectively. We also refer to [21].

Our framework covers an Assouad dichotomy result for fractional $(s, p, \beta)$-Hardy inequalities with $X=\mathbf{R}^{d}$, see the following Corollary 3 .

Corollary 3. Let $p, s, \beta$ be real numbers so that $0<p<\infty$ and $d+s p \geq 0$. Suppose $D \neq \emptyset$ is a proper $\kappa$-plump open set in $\mathbf{R}^{d}$ so that either condition $(T)$ or condition $(F)$ holds:

(T) $\overline{\operatorname{dim}}_{A}(\partial D)<d-s p+\beta$ and $D$ is unbounded;

(F) $\underline{\operatorname{dim}}_{A}(\partial D)>d-s p+\beta$, and $D$ is bounded or $\partial D$ is unbounded.

Then $D$ admits an $(s, p, \beta)$-Hardy inequality, i.e., inequality (1.3) holds for every $u \in C_{0}^{\infty}(D)$ with a constant $c>0$ independent of $u$.

This corollary follows from Theorem 1 with the aid of Example 3.3. As an illustration of this result, we may consider the domain $D \subset \mathbf{R}^{2}$ bounded by the Koch snowflake. It is a domain with a property $\underline{\operatorname{dim}}_{A}(\partial D)=\log 4 / \log 3$. Since $D$ is also $\kappa$ plump, Corollary 3 does apply. In the 'thin case' we may, e.g., consider the unbounded domain $G:=\mathbf{R}^{d} \backslash \bar{D}$. Now $G$ is $\kappa$-plump and it satisfies $\overline{\operatorname{dim}}_{A}(\partial G)=\log 4 / \log 3$. Let us note that [7, Theorem 1.1], apart from the case (T2) for $\alpha>1$, is a special case of Corollary 3. We would also like to note that we do not need to assume the positivity of $s$ in Corollary 3 .

Let us comment on the cases $(\mathrm{T})$ and $(\mathrm{F})$ in Corollary 3. Focusing on the case (T) first, recall that $\overline{\operatorname{dim}}_{A}(\partial D)=d-1$ for a Lipschitz domain $D$. The unboundedness of $D$ cannot be removed, at least if $0<s<1$, in which case a bounded Lipschitz domain satisfies an $(s, p, 0)$-Hardy inequality if and only if $s p>1$, [7]. Certain non-homogeneous $(s, p, 0)$-Hardy inequalities remain valid for John domains $D$ with $\overline{\operatorname{dim}}_{A}(\partial D)<d-s p,[18]$. Therein (T) with $\beta=0$ is formulated in terms of a certain Aikawa dimension which equals to the upper Assouad dimension in Euclidean spaces, see [25]. Recalling that John domains are both bounded and $\kappa$-plump, we may conclude that our framework provides a far-reaching generalisation of the aforementioned non-homogeneous results to the case of unbounded open sets.

Moving on to the case (F) with 'fat' boundary, let us first formulate an illustrative, but more restrictive, version of Corollary 3. We refer to $\S 4.4$ for the relevant definitions. 
Corollary 4. Let $p, s, \beta$ be real numbers so that $1<p<\infty, 0<s p-\beta<d$, and $d+s p \geq 0$. Suppose $D$ is a $\kappa$-plump open set in $\mathbf{R}^{d}$ such that $\partial D$ is $(s-\beta / p, p)$ uniformly fat (-locally uniformly fat, if $D$ is bounded). Then $D$ admits an $(s, p, \beta)$ Hardy inequality.

This corollary is a consequence of Corollary 3 and Propositions 8 and 9. Unlike in the case of inequality (1.4) with $\beta=0$ and $s=1$, the $(s, p)$-uniform fatness of $\partial D$ (let alone $D^{c}$ ) is not a sufficient condition for an open set $D$ to admit an $(s, p, 0)$ Hardy inequality (at least) in the case of $0<s p \leq 1$. This 'non-local obstruction' is recognised and addressed in [16]. It affects the fractional Hardy inequalities studied in [10], where $D^{c}$ is assumed to be $(s, p)$-uniformly fat and, as a conclusion, on the right hand side of (1.3) one then has integration over $\mathbf{R}^{d} \times \mathbf{R}^{d}$.

Suppose that $D$ is an open set whose boundary is $(s, p)$-uniformly fat (locally uniformly fat, if $D$ is bounded). It is an interesting question, what additional conditions are sufficient for $D$ to admit an $(s, p, 0)$-Hardy inequality. To this end, we improve [16, Corollary 1.4] where uniformity, [29, 36], of a domain $D$ is shown to be a sufficient additional condition. Indeed, by Corollary 4, we may replace uniformity with the significantly weaker $\kappa$-plumpness. Let us remark that [16, Theorem 4.1], stated in terms of a 'visibility condition on the boundary', still covers some other cases where our results do not apply, e.g., certain domains with outward cusps.

The structure of this paper is as follows. In $\S 2$ we define both the lower and upper Assouad dimension, and the notion of $\kappa$-plumpness. We also present other basic notation. Our main result is Theorem 5, stated and proven in $\S 3$. There we also define classes WLSC and WUSC of functions $\phi$ and a condition $\operatorname{DC}(a, \gamma, d)$ for open sets $D$. The latter condition is further clarified in $\S 4$ and $\S 5$, where we study the cases of 'fat' and 'thin' boundaries in terms of uniform fatness, and the lower and upper Assouad dimension.

Acknowledgment. Research is supported by the DFG through SFB-701 'Spectral Structures and Topological Methods in Mathematics'. Part of the research was done while the second author was visiting University of Bielefeld, and he would like to thank B. Dyda and M. Kaßmann for their hospitality. The first author was supported in part by the NCN grant 2012/07/B/ST1/03356. The authors would like to thank K. Bogdan, T. Grzywny and J. Lehrbäck for helpful discussions and preprints of [5] and [23].

\section{Assouad dimensions and plumpness}

We recall the lower and upper Assouad dimensions of a set $\emptyset \neq E \subset \mathbf{R}^{d}$, [19]. The lower Assouad dimension measures the 'fatness' of a set $E$, whereas the upper one measures how 'thin' a set $E$ is. The upper Assouad dimension is often called Assouad dimension, a notion tracing back to [2] and even [6]. We refer to [19, 28] for further information and other results.

Definition 2.1. Consider all $\lambda \geq 0$ for which there is $C>0$ so that, if $0<r<$ $R<2 \operatorname{diam}(E)$ and $x \in E$, then at least $C(R / r)^{\lambda}$ balls - centred in $E$ and of radius $r$-are needed to cover $B(x, R) \cap E$. The supremum of all such $\lambda$ is called the lower Assouad dimension of $E$ and it is denoted by $\underline{\operatorname{dim}}_{A}(E)$.

Definition 2.2. Consider all $\lambda \geq 0$ for which there is $C>0$ so that, if $0<r<$ $R<2 \operatorname{diam}(E)$ and $x \in E$, then we can cover $E \cap B(x, R)$ by at most $N \leq C(R / r)^{\lambda}$ 
balls $B_{1}, \ldots, B_{N}$ such that each $B_{j}$ is centred in $E$ and has radius $r$. We call the infimum of all such $\lambda$ the upper Assouad dimension of $E$, and write it as $\overline{\operatorname{dim}}_{A}(E)$.

We also recall a geometric notion from [36]. See also [30].

Definition 2.3. A set $A \subset \mathbf{R}^{d}$ is $\kappa$-plump with $\kappa \in(0,1)$ if, for each $0<r<$ $\operatorname{diam}(A)$ and each $x \in \bar{A}$, there is $z \in \bar{B}(x, r)$ such that $B(z, \kappa r) \subset A$.

Here is other notation; $(X, \rho, \mu)$ is a metric measure space, and we denote

$$
\delta_{x}=\operatorname{dist}\left(x, D^{c}\right)
$$

with $D^{c}=X \backslash D$. The open ball centred at $x \in X$ and of radius $r>0$ is denoted by $B(x, r) \subset X$. The boundary of set $A$ is written as $\partial A, \bar{A}$ denotes the closure of $A$, and $|A|$ is the Lebesgue measure of a measurable set $A \subset \mathbf{R}^{d}$. For a proper open set $D \subset \mathbf{R}^{d}$, we fix its Whitney decomposition $\mathcal{W}(D)$, and write $\mathcal{W}_{m}(D)$ for the family of Whitney cubes with side length $2^{-m}, m \in \mathbf{Z}$. If $Q \in \mathcal{W}(D)$, then

$$
\operatorname{diam}(Q) \leq \operatorname{dist}(Q, \partial D) \leq 4 \operatorname{diam}(Q) .
$$

For other properties of Whitney cubes we refer to [35, VI.1].

\section{Main result}

We state and prove our main result. For definition of conditions $\operatorname{DC}(a, \gamma, d)$, $\operatorname{WLSC}(\eta, 0, H)$ and $\operatorname{WUSC}(\eta, 0, H)$, we refer to $\S 3.1$ and $\S 3.2$. The proof of Theorem 5 is taken up in $\S 3.3$.

Theorem 5. Suppose that a proper open set $D \subset X$ satisfies $\operatorname{DC}(a, \gamma, d)$ with $a \in(0, \infty) \backslash\{1\}$. Moreover, suppose that for some $H \in(0,1]$, either $a \in(0,1), \eta+$ $\gamma-d>0$ and $\phi \in \operatorname{WLSC}(\eta, 0, H)$, or $a>1, \eta+\gamma-d<0$ and $\phi \in \operatorname{WUSC}\left(\eta, 0, H^{-1}\right)$. Then for any $0<p<\infty$ there exist constants $c$ and $R>0$ such that

$$
\int_{D} \frac{|u(x)|^{p}}{\phi\left(\delta_{x}\right)} \mu(d x) \leq c \int_{D} \int_{D \cap B\left(x, R \delta_{x}\right)} \frac{|u(x)-u(y)|^{p}}{\phi\left(\delta_{x}\right) \delta_{x}^{d}} \mu(d y) \mu(d x)
$$

for all measurable functions $u$ for which the left hand side is finite.

3.1. Assumptions on a function $\phi$. We adopt the notion of a global weak lower (or upper) scaling condition (WLSC or WUSC for short) from [5, Section 3]. In our case, the middle parameter in $\operatorname{WLSC}(\cdot, 0, \cdot)$ and $\operatorname{WUSC}(\cdot, 0, \cdot)$ is always zero and could be therefore omitted, but we prefer to keep the original notation. We formulate these conditions in an equivalent way, which is more convenient for our purposes than the original formulation.

Definition 3.1. Let $\eta \in \mathbf{R}$ and $H \in(0,1]$. We say that a function $\phi:(0, \infty) \rightarrow$ $(0, \infty)$ satisfies global WLSC $(\eta, 0, H)$ (resp., $\left.\operatorname{WUSC}\left(\eta, 0, H^{-1}\right)\right)$ and write $\phi \in \mathrm{WLSC}$ $(\eta, 0, H)\left(\phi \in \operatorname{WUSC}\left(\eta, 0, H^{-1}\right)\right)$, if

$$
\phi(s t) \geq H t^{\eta} \phi(s), \quad s>0,
$$

for every $t \geq 1$ (resp., for every $t \in(0,1]$ ).

Remark 3.2. If the domain $D$ in Theorem 5 is bounded, then it suffices to assume (3.2) for all $s, s t<\operatorname{diam}(D)$.

Example 3.3. Function $\phi(x)=x^{\eta}$ satisfies $\operatorname{WLSC}(\eta, 0,1)$ and $\operatorname{WUSC}(\eta, 0,1)$ whenever $\eta \in \mathbf{R}$. 
Example 3.4. Suppose that $\phi:(0, \infty) \rightarrow(0, \infty)$ is a regularly varying function at origin of index $\rho_{0}$, a regularly varying function at infinity of index $\rho_{\infty}$, and is bounded and bounded away from zero on every compact subset of $(0, \infty)$. If $\rho_{0}>\eta$ and $\rho_{\infty}>\eta$, then $\phi \in \operatorname{WLSC}(\eta, 0, H)$ for some $H \in(0,1]$, and if $\rho_{0}<\eta$ and $\rho_{\infty}<\eta$, then $\phi \in \operatorname{WUSC}\left(\eta, 0, H^{-1}\right)$ for some $H \in(0,1]$. These follow from Potter's theorem [3, Theorem 1.5.6].

We note that if, say, $a<1, \rho_{0} \geq \eta, \rho_{\infty} \geq \eta$, and if $\eta+\gamma-d>0$ and the assumptions on domain in Theorem 5 hold, then also the assertion (3.1) holds. Indeed, for every $\varepsilon>0$ function $\phi$ satisfies $\operatorname{WLSC}\left(\eta-\varepsilon, 0, H_{\varepsilon}\right)$ with some constant $H_{\varepsilon} \in(0,1]$, hence by taking $\varepsilon>0$ small enough we still have $(\eta-\varepsilon)+\gamma-d>0$.

To have more concrete examples, let us note that functions

$$
\phi_{1}(x)=x^{\alpha}+x^{\beta}, \quad \phi_{2}(x)=x^{\eta}(1+|\log x|)^{\beta}
$$

are regularly varying both at the origin (of indices $\min (\alpha, \beta)$ and $\eta$, respectively) and at infinity (of indices $\max (\alpha, \beta)$ and $\eta$, respectively).

Example 3.5. Functions $\phi$ are not confined to regularly varying functions. Indeed, $\phi(x)=x^{\eta} e^{x}$ satisfies $\operatorname{WLSC}(\eta, 0,1)$, but is not regularly varying at infinity.

3.2. Assumption $\operatorname{DC}(\boldsymbol{a}, \gamma, \boldsymbol{d})$ on open sets. In what follows we assume that $D$ is an open set in a metric measure space $(X, \rho, \mu)$.

Definition 3.6. We say that $D$ satisfies a domain condition $\operatorname{DC}(a, \gamma, d)$ where $\gamma \in \mathbf{R}, d>0, a>0, a \neq 1$, if there exist $M>0$ and (possibly empty) families $\mathcal{B}^{(n)}=\left\{B_{j}^{(n)}\right\}$ of subsets of $D$ indexed by $n \in \mathbf{Z}$ such that the following conditions (B1)-(B4) hold.

(B1) $D=\cup_{j, n} B_{j}^{(n)}$ and each $x \in D$ belongs to at most $M$ sets $B_{j}^{(n)}$.

(B2) For any $B_{j}^{(n)}$ we have

$$
M^{-1} a^{n} \leq \delta_{x} \leq M a^{n}, \quad x \in B_{j}^{(n)}, \quad M^{-1} a^{n d} \leq \mu\left(B_{j}^{(n)}\right) \leq M a^{n d} .
$$

(B3) For any $B_{j}^{(n)}$ and any integer $k>M$, there exists a nonempty finite set $V\left(B_{j}^{(n)}, k\right)$ of indices so that, for each $i \in V\left(B_{j}^{(n)}, k\right)$,

$$
\sup \left\{\rho(x, y): x \in B_{j}^{(n)} \text { and } y \in B_{i}^{(n+k)}\right\} \leq \begin{cases}M a^{n}, & \text { if } a<1 \\ M a^{n+k}, & \text { if } a>1 .\end{cases}
$$

(B4) For each $n \in \mathbf{Z}$ and $k>M$,

$$
\sup _{i} \sum_{j: i \in V\left(B_{j}^{(n)}, k\right)} \frac{1}{\sharp V\left(B_{j}^{(n)}, k\right)} \leq M a^{k \gamma} .
$$

Remark 3.7. The slightly technical Definition 3.6 allows a unified treatment of fractional Hardy inequalities in different cases. In the Euclidean spaces, we usually take Whitney cubes of roughly the same size as the sets $B_{j}^{(n)}$ and then conditions $(B 1)$ and $(B 2)$ are immediate, we refer to $\S 4.1$ and $\S 5.1$ for examples. Formulating the definition using these general sets rather than Whitney cubes gives us some flexibility, see Examples 3.8 and 3.9 for an illustration. The last two conditions (B3) and (B4) specify the relationship between the layers

$$
D_{n}:=D \cap\left\{x: M^{-1} a^{n} \leq \delta_{x} \leq M a^{n}\right\}
$$


and

$$
D_{n+k}:=D \cap\left\{x: M^{-1} a^{n+k} \leq \delta_{x} \leq M a^{n+k}\right\}
$$

for large values of $k$. Namely, in a 'neighbourhood' of each $B_{j}^{(n)} \subset D_{n}$ there should be 'sufficiently many' sets $B_{i}^{(n+k)} \subset D_{n+k}$; the number $\gamma$ describes this quantitatively.

Below we provide some illustrative examples of sets satisfying condition $\operatorname{DC}(a, \gamma, d)$. In the two examples $X=\mathbf{R}^{d}$ with the Euclidean distance, in which case $\delta_{x}=$ $\operatorname{dist}(x, \partial D)$ for all $x \in D$. Moreover, $\mu$ is the Lebesgue measure.

Example 3.8. Set $D=\mathbf{R}^{d} \backslash\{0\}$ satisfies condition $\operatorname{DC}(a, \gamma, d)$ with $a=2, \gamma=0$ and

$$
M=2 \vee\left(1-2^{-d}\right)|B(0,1)| \vee \frac{1}{\left(1-2^{-d}\right)|B(0,1)|}
$$

Indeed, one may take $B_{1}^{(n)}:=B\left(0,2^{n}\right) \backslash B\left(0,2^{n-1}\right)$. That is, for each $n$ there is exactly one set $B_{j}^{(n)}$, namely one with $j=1$. Then $V\left(B_{j}^{(n)}, k\right)=\{1\}$ in (B3).

Example 3.9. Set $D=\mathbf{R}^{d} \backslash\{0\}$ satisfies condition $\operatorname{DC}(a, \gamma, d)$ with $a=\frac{1}{2}$, $\gamma=0$ and

$$
M=2 \vee\left(1-2^{-d}\right)|B(0,1)| \vee \frac{1}{\left(1-2^{-d}\right)|B(0,1)|} .
$$

Indeed, one may take $B_{1}^{(n)}:=B\left(0,2^{-n}\right) \backslash B\left(0,2^{-n-1}\right)$ and $V\left(B_{j}^{(n)}, k\right)=\{1\}$ in (B3).

3.3. Proof of Theorem 5. Let us write

$$
q=2^{p+1} M^{4+2|\eta|} H^{-1} a^{k(\eta+\gamma-d)}, \quad R=1+M^{2}\left(1 \vee a^{k}\right), \quad S=2^{p+1} a^{-k d} M^{d+1},
$$

where $k>M$ is chosen such that $q<1$ and $a^{k} \vee a^{-k}>M^{2}$.

We fix a function $u$ for which the left hand side of (3.1) is finite, and define a set

$$
F=\left\{x \in D:|u(x)|^{p}>S \delta_{x}^{-d} \int_{D \cap B\left(x, R \delta_{x}\right)}|u(x)-u(y)|^{p} \mu(d y)\right\} .
$$

Let us first observe that, for $x \in D \backslash F$,

$$
\frac{|u(x)|^{p}}{\phi\left(\delta_{x}\right)} \leq S \int_{D \cap B\left(x, R \delta_{x}\right)} \frac{|u(x)-u(y)|^{p}}{\phi\left(\delta_{x}\right) \delta_{x}^{d}} \mu(d y) .
$$

Note that if the set $F$ were empty, we would be already done. have

At this stage we fix $n$ and claim that, for $x \in F \cap B_{j}^{(n)}$ and $i \in V\left(B_{j}^{(n)}, k\right)$, we

$$
\mu\left(\left\{y \in B_{i}^{(n+k)}: \frac{1}{2}|u(x)| \leq|u(y)| \leq \frac{3}{2}|u(x)|\right\}\right) \geq \frac{1}{2} \mu\left(B_{i}^{(n+k)}\right) .
$$

Suppose (3.4) fails. By our choice of $R$ and conditions (B2) and (B3), $B_{i}^{(n+k)} \subset$ $D \cap B\left(x, R \delta_{x}\right)$. Thus, we have

$$
\begin{aligned}
& \int_{D \cap B\left(x, R \delta_{x}\right)}|u(x)-u(y)|^{p} \mu(d y) \geq \int_{B_{i}^{(n+k)}}|u(x)-u(y)|^{p} \mu(d y) \\
& \geq \frac{1}{2} \mu\left(B_{i}^{(n+k)}\right) \cdot 2^{-p}|u(x)|^{p} \geq 2^{-p-1} a^{k d} M^{-d-1} \delta_{x}^{d}|u(x)|^{p}=S^{-1} \delta_{x}^{d}|u(x)|^{p},
\end{aligned}
$$

which contradicts $x \in F$. Thus inequality (3.4) holds as claimed. 
Let us record the following estimates for $B_{i}^{(n+k)} \in \mathcal{B}^{(n+k)}$ and $B_{j}^{(n)} \in \mathcal{B}^{(n)}$. By condition (B2), $\mu\left(B_{j}^{(n)}\right) \leq M^{2} a^{-k d} \mu\left(B_{i}^{(n+k)}\right)$, moreover, for $x \in B_{j}^{(n)}$ and $y \in B_{i}^{(n+k)}$ it holds $M^{2} a^{-k} \delta_{y} \geq \delta_{x} \geq M^{-2} a^{-k} \delta_{y}$. Hence, by condition (3.2)

$$
\phi\left(\delta_{x}\right)=\phi\left(\delta_{y} \frac{\delta_{x}}{\delta_{y}}\right) \geq H\left(\frac{\delta_{x}}{\delta_{y}}\right)^{\eta} \phi\left(\delta_{y}\right) \geq H M^{-2|\eta|} a^{-k \eta} \phi\left(\delta_{y}\right) .
$$

Here we need to ensure that $\frac{\delta_{x}}{\delta_{y}}<1$ in the case when $a>1$ and that $\frac{\delta_{x}}{\delta_{y}}>1$ in the case when $a<1$. But these are satisfied since, by assumption, $a^{k} \vee a^{-k}>M^{2}$, i.e., $k$ is large enough. By the above estimate and inequality (3.4) we obtain

$$
\begin{aligned}
\int_{F \cap B_{j}^{(n)}} \frac{|u(x)|^{p}}{\phi\left(\delta_{x}\right)} \mu(d x) & \leq \mu\left(B_{j}^{(n)}\right) \sup _{x \in F \cap B_{j}^{(n)}} \frac{|u(x)|^{p}}{\phi\left(\delta_{x}\right)} \\
& \leq \frac{2^{p+1} M^{2} a^{-k d}}{\sharp V\left(B_{j}^{(n)}, k\right)} \sum_{i \in V\left(B_{j}^{(n)}, k\right)} \int_{B_{i}^{(n+k)}} \frac{|u(y)|^{p}}{H M^{-2|\eta|} a^{-k \eta} \phi\left(\delta_{y}\right)} \mu(d y) .
\end{aligned}
$$

By summing over all $j$ and applying condition (B4),

$$
\begin{aligned}
& \sum_{j} \int_{F \cap B_{j}^{(n)}} \frac{|u(x)|^{p}}{\phi\left(\delta_{x}\right)} \mu(d x) \\
& \leq 2^{p+1} M^{2+2|\eta|} H^{-1} a^{k(\eta-d)} \sup _{i} \sum_{j: i \in V\left(B_{j}^{(n)}, k\right)} \frac{1}{\sharp V\left(B_{j}^{(n)}, k\right)} \sum_{i} \int_{B_{i}^{(n+k)}} \frac{|u(y)|^{p}}{\phi\left(\delta_{y}\right)} \mu(d y) \\
& \leq 2^{p+1} M^{3+2|\eta|} H^{-1} a^{k(\eta+\gamma-d)} \sum_{i} \int_{B_{i}^{(n+k)}} \frac{|u(y)|^{p}}{\phi\left(\delta_{y}\right)} \mu(d y),
\end{aligned}
$$

and after summing over all $n$

$$
\int_{F} \frac{|u(x)|^{p}}{\phi\left(\delta_{x}\right)} \mu(d x) \leq q \int_{D} \frac{|u(y)|^{p}}{\phi\left(\delta_{y}\right)} \mu(d y)
$$

Recall that $q<1$. Hence, by finiteness of the left hand side of (3.1),

$$
\int_{F} \frac{|u(x)|^{p}}{\phi\left(\delta_{x}\right)} \mu(d x) \leq \frac{q}{1-q} \int_{D \backslash F} \frac{|u(y)|^{p}}{\phi\left(\delta_{y}\right)} \mu(d y) .
$$

This estimate and inequality (3.3) finish the proof.

3.4. Proof of Corollary 2. We use Potter's theorem [3, Theorem 1.5.6] to replace $\phi\left(\delta_{x}\right)$ by $c \phi(|x-y|)$ in the denominator, with $c=c(R, \phi)$. The assumption $0<\rho_{0}, \rho_{\infty}$ is used here. The result follows now from Theorem 5 and Examples 3.4, 3.8 and 3.9 .

\section{Fat boundary}

We prove a domain condition while assuming that the boundary of the open set is sufficiently 'fat' in terms of the lower Assouad dimension. Then we study the relation between lower Assouad dimension and uniform fatness.

Proposition 6. Suppose $D \neq \emptyset$ is a proper $\kappa$-plump open set in $\mathbf{R}^{d}$ such that $D$ is bounded or $\partial D$ is unbounded. Then $D$ satisfies $\operatorname{DC}(a, \lambda, d)$ if $a=1 / 2$ and either 
$0<\lambda<\underline{\operatorname{dim}}_{A}(\partial D)$ or $\lambda=0$. Moreover, the associated constant $M$ depends only on $d, \kappa, \lambda$ and the constant $C$ appearing in (F1) below.

Under the assumptions of Proposition 6, the following two conditions hold.

(F1) There is a constant $C>0$ as follows. Let $0<r<R<2 \operatorname{diam}(\partial D)$ and $x \in \partial D$. Suppose that $B_{1}, \ldots, B_{N}$ is a cover of $B(x, R) \cap \partial D$ by balls $B_{j}=$ $B\left(\omega_{j}, r\right)$ with $\omega_{j} \in \partial D$ for $j=1, \ldots, N$. Then $N \geq C(R / r)^{\lambda}$.

(F2) for each $0<r<\operatorname{diam}(D)$ and each $x \in \partial D$, there is $z \in \bar{B}(x, r)$ so that $B(z, \kappa r) \subset D$.

4.1. Construction of families $\mathcal{B}^{(n)}$. We define a constant

$$
\tau=\left(\frac{15 \sqrt{d}}{\kappa}\right)^{d}>1
$$

For a given $n \in \mathbf{Z}$ and $a \in\left\{\frac{1}{2}, 2\right\}$, we define

$$
\mathcal{B}^{(n)}:=\mathcal{B}_{1 / 2}^{(n)}, \quad \mathcal{B}_{a}^{(n)}:=\left\{B_{j}^{(n)}\right\}:=\left\{Q \in \mathcal{W}(D): \tau^{-1} \leq a^{-n d}|Q| \leq \tau\right\} .
$$

Recall that $\mathcal{W}(D)$ stands for a Whitney decomposition of $D$. In particular, by inequalities (2.1), for any $x \in B_{j}^{(n)} \in \mathcal{B}_{a}^{(n)}$,

$$
\tau^{-1 / d} a^{n} \leq \delta_{x}=\operatorname{dist}(x, \partial D) \leq 5 \sqrt{d} \tau^{1 / d} a^{n} .
$$

Observe also that a given Whitney cube $Q \in \mathcal{W}(D)$ may belong to at most $1+$ $2 d^{-1} \log _{2} \tau$ families $\mathcal{B}^{(n)}=\mathcal{B}_{a}^{(n)}$ indexed by $n \in \mathbf{Z}$. Let us denote by $x_{j}^{(n)}$ the midpoint of $B_{j}^{(n)}$. For later purposes we fix, once and for all, any point $y_{j}^{(n)} \in \partial D$ for which

$$
\left|x_{j}^{(n)}-y_{j}^{(n)}\right|=\operatorname{dist}\left(x_{j}^{(n)}, \partial D\right) .
$$

4.2. Families $\boldsymbol{V}\left(\boldsymbol{B}_{\boldsymbol{j}}^{(\boldsymbol{n})}, \boldsymbol{k}\right)$ for $\boldsymbol{k}$ large. If $D$ is unbounded, we construct families $V\left(B_{j}^{(n)}, k\right)$ for $k>3$. If $D$ is bounded, then we construct these families for $k>3 \vee \log _{2}\left(5 \tau^{1 / d}\right)$.

Let us fix $B_{j}^{(n)} \in \mathcal{B}^{(n)}$, and define $E:=B\left(y_{j}^{(n)}, 2^{-n}\right) \cap \partial D$. By the $5 r$-covering theorem, see for instance [31, p. 23], there are points $\omega_{1}, \ldots, \omega_{N} \in E$ such that the balls $B_{m}:=B\left(\omega_{m}, 2^{-n-k}\right)$ are disjoint and $E$ is covered by the union of balls $5 B_{m}$, $m=1, \ldots, N$. Let us estimate the number $N=: N_{j}^{(n, k)}$ of these balls;

Lemma 7. We have $N_{j}^{(n, k)} \geq C 5^{-\lambda} \tau^{-\lambda / d} 2^{k \lambda}$.

Proof. First consider the case when $D$ is unbounded. Since $k>3$, we find that

$$
r:=5 \cdot 2^{-n-k}<2^{-n}=: R .
$$

Recall that the balls $5 B_{m}=B\left(\omega_{m}, r\right)$ cover the set $E=B\left(y_{j}^{(n)}, R\right) \cap \partial D$. By condition (F1), we find that $N \geq C(R / r)^{\lambda}=C 5^{-\lambda} 2^{k \lambda}$. The bounded case is similar, and we use the facts that $k>3 \vee \log _{2}\left(5 \tau^{1 / d}\right)$ and $\operatorname{diam}(\partial D) \geq \operatorname{diam}(D)$.

The next step is to use the plumpness condition $(\mathrm{F} 2)$ in order to locate a sufficiently large cube inside each $B_{m}$. Namely, for each $m=1, \ldots, N$, there is $z_{m} \in \bar{B}\left(\omega_{m}, 2^{-n-k} / 3\right)$ such that

$$
B\left(z_{m}, \kappa 2^{-n-k} / 3\right) \subset D .
$$


Let us consider a Whitney cube $Q_{m} \in \mathcal{W}(D)$ for which $z_{m} \in Q_{m}$. By inequalities (2.1), we have $Q_{m} \subset B_{m}$. Moreover,

$$
\kappa 2^{-n-k} / 3 \leq \operatorname{dist}\left(z_{m}, \partial D\right) \leq 5 \operatorname{diam}\left(Q_{m}\right) \leq 5 \operatorname{dist}\left(z_{m}, \partial D\right) \leq 5 \cdot 2^{-n-k} / 3 .
$$

Hence, by our definition (4.1) of $\tau$, we obtain

$$
\tau^{-1} \leq 2^{d(n+k)}\left|Q_{m}\right| \leq \tau .
$$

That is, cube $Q_{m} \subset B_{m}$ belongs to $\mathcal{B}^{(n+k)}$. Since the balls $B_{m}, m=1, \ldots, N$, are disjoint, also the cubes $Q_{m}$ are disjoint. Hence, the indexing set

$$
V\left(B_{j}^{(n)}, k\right)=\left\{i: B_{i}^{(n+k)}=Q_{m} \text { for some } m=1, \ldots, N_{j}^{(n, k)}\right\}
$$

contains exactly $N_{j}^{(n, k)}$ indices.

4.3. Proof of Proposition 6. We focus on conditions (B3) and (B4), as the remaining conditions are clearly satisfied. Let us fix $B_{j}^{(n)}$ and $k$ large enough so that $V\left(B_{j}^{(n)}, k\right)$ is defined. Let us consider $i \in V\left(B_{j}^{(n)}, k\right)$, and two given points $x \in B_{j}^{(n)}$ and $y \in B_{i}^{(n+k)}$. Using the notation above, we have $B_{i}^{(n+k)}=Q_{m} \subset B_{m}$ for some $m=1, \ldots, N_{j}^{(n, k)}$. Thus,

$$
\begin{aligned}
|x-y| & \leq\left|x-x_{j}^{(n)}\right|+\left|x_{j}^{(n)}-y_{j}^{(n)}\right|+\left|y_{j}^{(n)}-\omega_{m}\right|+\left|\omega_{m}-y\right| \\
& <\operatorname{diam}\left(B_{j}^{(n)}\right)+\operatorname{dist}\left(x_{j}^{(n)}, \partial D\right)+2^{-n}+2^{-n-k} \leq 8 \sqrt{d} \tau^{1 / d} 2^{-n} .
\end{aligned}
$$

This is condition (B3). A particular consequence of this estimate is the following. We fix a cube $B_{i}^{(n+k)}$ and a point $y$ therein. Then, if $B_{j}^{(n)} \in \mathcal{B}^{(n)}$ is such that $i \in V\left(B_{j}^{(n)}, k\right)$,

$$
B_{j}^{(n)} \subset B\left(y, 8 \sqrt{d} \tau^{1 / d} 2^{-n}\right) .
$$

Since the interiors of cubes in $\mathcal{B}^{(n)}$ are disjoint, we find that there are at most

$$
\frac{\left(16 \sqrt{d} \tau^{1 / d} 2^{-n}\right)^{d}}{\tau^{-1} 2^{-n d}}=(16 \sqrt{d})^{d} \tau^{2}
$$

cubes $B_{j}^{(n)}$ subject to the conditions above. By using this fact, we may now deduce the remaining estimate as follows; For a fixed $i$,

$$
\sum_{j: i \in V\left(B_{j}^{(n)}, k\right)} \frac{1}{\sharp V\left(B_{j}^{(n)}, k\right)}=\sum_{j: i \in V\left(B_{j}^{(n)}, k\right)} \frac{1}{N_{j}^{(n, k)}} \leq(16 \sqrt{d})^{d} \tau^{2+\lambda / d} C^{-1} 5^{\lambda} 2^{-k \lambda} .
$$

This is condition (B4).

4.4. Lower Assouad dimension and uniform fatness. We provide a useful connection between the lower Aikawa dimension and (local) uniform fatness. For further discussion, we refer to [19]. Uniform fatness is usually defined in terms of Riesz capacities, [1, 26]. In case of closed sets, there is an equivalent definitionin terms of Hausdorff content - that we adopt. This equivalence is based on the self-improving properties of closed uniformly fat sets, [16].

Recall that the $\lambda$-Hausdorff content of a set $E \subset \mathbf{R}^{d}$ is

$$
\mathcal{H}_{\infty}^{\lambda}(E)=\inf \left\{\sum_{i=1}^{\infty} r_{i}^{\lambda}: E \subset \bigcup_{i=1}^{\infty} B\left(x_{i}, r_{i}\right), r_{i}>0\right\} .
$$


As is easily seen, we may allow also finite coverings in the infimum above. Let $1<p<\infty$ and $0<s<d / p$. We say that the boundary $\partial D$ is $(s, p)$-uniformly fat, if there is $d-s p<\lambda \leq d$ and a constant $C>0$ such that

$$
\mathcal{H}_{\infty}^{\lambda}(B(x, R) \cap \partial D) \geq C R^{\lambda}
$$

for all $x \in \partial D$ and $R>0$.

Note that $\partial D$ and $D$ have to be unbounded if the boundary is $(s, p)$-uniformly fat. Remark 2.3 in [19] shows that $\underline{\operatorname{dim}}_{A}(\partial D)$ is the supremum of all $\lambda \geq 0$ for which (4.2) holds for every $x \in \partial D$ and $0<R<\operatorname{diam}(\partial D)$. Below, for the convenience of the reader, we provide a detailed treatment of certain consequences of this statementthat are needed for Corollary 4.

Proposition 8. Suppose $D$ is an open set in $\mathbf{R}^{d}$ so that $\partial D$ is $(s, p)$-uniformly fat for $1<p<\infty$ and $0<s<d / p$. Then $D$ satisfies condition (F1) for some $d-s p<\lambda \leq d$ and, as a consequence, we have a strict inequality $\underline{\operatorname{dim}}_{A}(\partial D)>d-s p$.

Proof. By assumption, there is $d-s p<\lambda \leq d$ and $C>0$ such that (4.2) holds for all $x \in \partial D$ and $R>0$. Let us fix $x \in \partial D$ and $0<r<R$. Suppose that $B_{1}, \ldots, B_{N}$ is a cover of $B(x, R) \cap \partial D$ by balls $B_{j}=B\left(\omega_{j}, r\right)$ with $\omega_{j} \in \partial D$. Then, by $(4.2)$,

$$
N r^{\lambda}=\sum_{j=1}^{N} r^{\lambda} \geq \mathcal{H}_{\infty}^{\lambda}(B(x, R) \cap \partial D) \geq C R^{\lambda} .
$$

Thus, $N \geq C(R / r)^{\lambda}$, as required.

As we have observed, uniform fatness is a convenient notion in case of unbounded open sets. In case of a bounded open set $D$ in $\mathbf{R}^{d}$, it is natural to assume that $\partial D$ is $(s, p)$-locally uniformly fat. That is, there is $d-s p<\lambda \leq d$ and a constant $C>0$ such that inequality (4.2) holds for all $x \in \partial D$ and $0<R<2 \operatorname{diam}(\partial D)<\infty$.

The following result is analogous to Proposition 8.

Proposition 9. Let $D$ be a bounded open set in $\mathbf{R}^{d}$ such that the boundary $\partial D$ is $(s, p)$-locally uniformly fat for $1<p<\infty$ and $0<s<d / p$. Then $D$ satisfies condition (F1) for some $d-s p<\lambda \leq d$ and, as a consequence, we have a strict inequality $\underline{\operatorname{dim}}_{A}(\partial D)>d-s p$.

Example 4.1. Consider the Koch snowflake domain $D \subset \mathbf{R}^{2}$. It is a bounded $\kappa$-plump domain and $\partial D$ is $(s, p)$-locally uniformly fat whenever $1<p<\infty$ and $2-\log 4 / \log 3<s p<2$, see e.g. [16].

\section{Thin boundary}

The main result in this section is the following.

Proposition 10. Let $D \neq \emptyset$ be an unbounded $\kappa$-plump open set in $\mathbf{R}^{d}, D \neq \mathbf{R}^{d}$. Then $D$ satisfies condition $\operatorname{DC}(a, \lambda, d)$ for $a=2$ and $\lambda>\overline{\operatorname{dim}}_{A}(\partial D)$. The associated constant $M$ depends only on $d, \kappa, \lambda$ and the constant $C$ appearing in (T1) below.

Before the proof, let us clarify the assumptions. Under the assumptions of Proposition 10, the following two conditions (T1) and (T2) hold.

(T1) there is a constant $C>0$ as follows. Assuming that $0<r<R$ and $x \in$ $\partial D$, there is a cover of $B(x, R) \cap \partial D$ by using balls $B\left(\omega_{j}, r\right)$ with $\omega_{j} \in \partial D$, 
$j=1, \ldots, N$, such that the number of these balls satisfies inequality $N \leq$ $C(R / r)^{\lambda}$.

(T2) for each $0<r$ and each $x \in \partial D$, there is a point $z \in \bar{B}(x, r)$ such that $B(z, \kappa r) \subset D$.

We note that in Definition 2.2 the restriction $R<2 \operatorname{diam}(E)$ may be removed, resulting in no such restriction in (T1).

5.1. Construction of families $\mathcal{B}^{(n)}$. Let $\tau>1$ be defined by (4.1). For $n \in \mathbf{Z}$, we define

$$
\mathcal{B}^{(n)}:=\mathcal{B}_{2}^{(n)}:=\left\{B_{j}^{(n)}\right\}:=\left\{Q \in \mathcal{W}(D): \tau^{-1} \leq 2^{-n d}|Q| \leq \tau\right\}
$$

For properties of cubes in these families and definition of $y_{j}^{(n)}$, see $\S 4.1$. Recall also that $\mathcal{W}(D)$ is a Whitney decomposition of $D$, we refer to $\S 2$.

5.2. Families $\boldsymbol{V}\left(\boldsymbol{B}_{\boldsymbol{j}}^{(\boldsymbol{n})}, \boldsymbol{k}\right)$ with $\boldsymbol{k}>\mathbf{0}$. Let us fix a cube $B_{j}^{(n)} \in \mathcal{B}^{(n)}$. By condition (T2), there is a point $z_{j}^{(n)} \in \bar{B}\left(y_{j}^{(n)}, 2^{n+k}\right)$ such that

$$
B\left(z_{j}^{(n)}, \kappa 2^{n+k}\right) \subset D .
$$

Observe how the unboundedness of $D$ is visible here, as $k>0$ is arbitrary. Let $Q_{j} \in \mathcal{W}(D)$ be a Whitney cube such that $z_{j}^{(n)} \in Q_{j}$. Then

$$
\kappa \cdot 2^{n+k} \leq \operatorname{dist}\left(z_{j}^{(n)}, \partial D\right) \leq 5 \operatorname{diam}\left(Q_{j}\right) \leq 5 \operatorname{dist}\left(z_{j}^{(n)}, \partial D\right) \leq 5 \cdot 2^{n+k} .
$$

By definition of (4.1) of $\tau$, we then have $Q_{j}=B_{i}^{(n+k)} \in \mathcal{B}^{(n+k)}$ for some index $i$. We define

$$
V\left(B_{j}^{(n)}, k\right)=\{i\} .
$$

5.3. Proof of Proposition 10. We need an auxiliary estimate analogous to [19, Lemma 4.3]; condition (T1) is our primary tool. For $m \in \mathbf{Z}, \omega \in \partial D$, and $R>0$, we denote

$$
\mathcal{W}_{m}(D ; B(\omega, R))=\left\{Q \in \mathcal{W}_{m}(D): Q \subset B(\omega, R)\right\}
$$

Lemma 11. Let $0<2^{-m} \leq R$, where $m \in \mathbf{Z}$, and let $\lambda>\overline{\operatorname{dim}}_{A}(\partial D)$. Then for every $\omega \in \partial D$,

$$
\sharp \mathcal{W}_{m}(D ; B(\omega, R)) \leq C(14 \sqrt{d})^{d+\lambda}\left(\frac{R}{2^{-m}}\right)^{\lambda},
$$

where $C$ is as in condition (T1).

Proof. Suppose $B_{1}, \ldots, B_{N}$ is a cover of $B(\omega, 6 \sqrt{d} R) \cap \partial D$ by balls $B_{j}=$ $B\left(\omega_{j}, 2^{-m}\right)$ that are centred in $\partial D$, see condition (T1). Consider a cube $Q \in$ $\mathcal{W}_{m}(D ; B(\omega, R))$, and fix a point $y_{Q} \in \partial D$ such that $\left|x_{Q}-y_{Q}\right|=\operatorname{dist}\left(x_{Q}, \partial D\right)$. Here $x_{Q}$ denotes the midpoint of $Q$. By inequalities (2.1) and the fact that $Q \subset B(\omega, R)$,

$$
\left|y_{Q}-\omega\right| \leq\left|y_{Q}-x_{Q}\right|+\left|x_{Q}-\omega\right|<5 \operatorname{diam}(Q)+R \leq 6 \sqrt{d} R .
$$

By the covering property, there is $j=j(Q)$ such that $y_{Q} \in B_{j}$. We can infer that

$$
\mathcal{W}_{m}(D ; B(\omega, R))=\bigcup_{j=1}^{N} \mathcal{Q}_{j}
$$


where $\mathcal{Q}_{j}=\left\{Q \in \mathcal{W}_{m}(D ; B(\omega, R)): y_{Q} \in B_{j}\right\}$. Let $Q \in \mathcal{Q}_{j}$. Then, for every $x \in Q$,

$$
\begin{aligned}
\left|x-\omega_{j}\right| & \leq\left|x-x_{Q}\right|+\left|x_{Q}-y_{Q}\right|+\left|y_{Q}-\omega_{j}\right| \\
& <\operatorname{diam}(Q)+\operatorname{dist}\left(x_{Q}, \partial D\right)+2^{-m} \leq 7 \sqrt{d} 2^{-m} .
\end{aligned}
$$

Since the interiors of cubes in the family $\mathcal{Q}_{j}$ are disjoint, there are at most

$$
\frac{\left|B\left(\omega_{j}, 7 \sqrt{d} 2^{-m}\right)\right|}{2^{-m d}} \leq(14 \sqrt{d})^{d}
$$

cubes in this family. Hence,

$$
\sharp \mathcal{W}_{m}(D ; B(\omega, R)) \leq \sum_{j=1}^{N} \sharp \mathcal{Q}_{j} \leq(14 \sqrt{d})^{d} N \leq C\left(\frac{6 \sqrt{d} R}{2^{-m}}\right)^{\lambda} \cdot(14 \sqrt{d})^{d} .
$$

This concludes the proof.

We are ready to prove the main result in this section.

Proof of Proposition 10. The properties (B1) and (B2) are clear. In order to verify condition (B3), let us fix $k>0$ and a cube $B_{j}^{(n)}$. Consider $i \in V\left(B_{j}^{(n)}, k\right)$, and points $x \in B_{j}^{(n)}$ and $z \in B_{i}^{(n+k)}$. Then, by the construction above,

$$
\begin{aligned}
|z-x| & \leq\left|z-z_{j}^{(n)}\right|+\left|z_{j}^{(n)}-y_{j}^{(n)}\right|+\left|y_{j}^{(n)}-x_{j}^{(n)}\right|+\left|x_{j}^{(n)}-x\right| \\
& \leq \operatorname{diam}\left(B_{i}^{(n+k)}\right)+2^{n+k}+\operatorname{dist}\left(x_{j}^{(n)}, \partial D\right)+\operatorname{diam}\left(B_{j}^{(n)}\right)<8 \sqrt{d} \tau^{1 / d} 2^{n+k} .
\end{aligned}
$$

This is condition (B3).

In order to verify the last condition (B4), we fix cubes $B_{i}^{(n+k)}$ and $B_{j}^{(n)}$ such that $i \in V\left(B_{j}^{(n)}, k\right)$. Then $\sharp V\left(B_{j}^{(n)}, k\right)=1$. Moreover,

$$
B_{j}^{(n)} \subset B\left(y_{i}^{(n+k)}, 13 \sqrt{d} \tau^{1 / d} 2^{n+k}\right) .
$$

Indeed, for any $x \in B_{j}^{(n)}$,

$$
\left|x-y_{i}^{(n+k)}\right| \leq\left|x-x_{i}^{(n+k)}\right|+\left|x_{i}^{(n+k)}-y_{i}^{(n+k)}\right|<13 \sqrt{d} \tau^{1 / d} 2^{n+k} .
$$

We still need another auxiliary estimate, namely, if $m \in \mathbf{Z}$ is such that $B_{j}^{(n)} \in$ $\mathcal{W}_{m}(D)$, then $\tau^{-1 / d} \leq 2^{m+n} \leq \tau^{1 / d}$. We can finally proceed as follows

$$
\begin{aligned}
\sum_{j: i \in V\left(B_{j}^{(n)}, k\right)} \frac{1}{\sharp V\left(B_{j}^{(n)}, k\right)} & =\sharp\left\{j: i \in V\left(B_{j}^{(n)}, k\right)\right\} \\
& =\sum_{m} \sharp\left\{j: i \in V\left(B_{j}^{(n)}, k\right) \text { and } B_{j}^{(n)} \in \mathcal{W}_{m}(D)\right\} \\
& \leq \sum_{m} \sharp \mathcal{W}_{m}\left(D ; B\left(y_{i}^{(n+k)}, 13 \sqrt{d} \tau^{1 / d} 2^{n+k}\right)\right) \\
& \leq \sum_{m} C(14 \sqrt{d})^{d+\lambda}\left(\frac{13 \sqrt{d} \tau^{1 / d} 2^{n+k}}{2^{-m}}\right)^{\lambda},
\end{aligned}
$$

where $m$ ranges over indices $-n-\log _{2} \tau^{1 / d} \leq m \leq-n+\log _{2} \tau^{1 / d}$. This yields condition (B4). 


\section{References}

[1] Adams, D. R., and L. I. HedBerg: Function spaces and potential theory. - Grundlehren Math. Wiss. 314, Springer-Verlag, Berlin, 1996.

[2] Assound, P.: Plongements lipschitziens dans $\mathbf{R}^{n}$. - Bull. Soc. Math. France 111:4, 1983, 429-448.

[3] Bingham, N. H., C. M. Goldie, and J. L. Teugels: Regular variation. - Encyclopedia Math. Appl. 27, Cambridge Univ. Press, Cambridge, 1989.

[4] Bogdan, K., and B. Dyda: The best constant in a fractional Hardy inequality. - Math. Nachr. 284:5-6, 2011, 629-638.

[5] Bogdan, K., T. Grzywny, and M. Ryznar: Density and tails of unimodal convolution semigroups. - arXiv:1305.0976, 2013.

[6] Bouligand, G.: Sur quelques points de topologie restreinte du premier ordre. - Bull. Soc. Math. France 56, 1928, 26-35.

[7] DydA, B.: A fractional order Hardy inequality. - Illinois J. Math. 48:2, 2004, 575-588.

[8] DyDA, B.: Fractional Hardy inequality with a remainder term. - Colloq. Math. 122:1, 2011, 59-67.

[9] Dyda, B., and R. L. FRAnk: Fractional Hardy-Sobolev-Maz'ya inequality for domains. Studia Math. 208:2, 2012, 151-166.

[10] Edmunds, D. E., R. Hurri-Syrjänen, and A. V. VÄHÄKangas: Fractional Hardy-type inequalities in domains with uniformly fat complement. - Proc. Amer. Math. Soc. 142:3, 2014, $897-907$.

[11] Filippas, S., L. Moschini, and A. Tertikas: Sharp trace Hardy-Sobolev-Maz'ya inequalities and the fractional Laplacian. - Arch. Ration. Mech. Anal. 208:1, 2013, 109-161.

[12] Frank, R. L., and R. SeIRINGer: Sharp fractional Hardy inequalities in half-spaces. - In: Around the research of Vladimir Maz'ya. I, Int. Math. Ser. (N. Y.) 11, 2010, 161-167.

[13] Grzywny, T.: On Harnack inequality and Hölder regularity for isotropic unimodal Lévy processes. - arXiv:1301.2441, 2013.

[14] Gurka, P., and B. OpIC: Sharp Hardy inequalities of fractional order involving slowly varying functions. - J. Math. Anal. Appl. 386:2, 2012, 728-737.

[15] Heinig, H. P., A. Kufner, and L.-E. Persson: On some fractional order Hardy inequalities. - J. Inequal. Appl. 1:1, 1997, 25-46.

[16] Ihnatsyeva, L., J. Lehrbäck, H. Tuominen, and A. V. VÄhäkangas: Fractional Hardy inequalities and visibility of the boundary. - arXiv:1305.4616, 2013.

[17] Innatsyeva, L., and A. V. VÄHÄKangas: Hardy inequalities in Triebel-Lizorkin spaces. Indiana Univ. Math. J. (to appear).

[18] Ihnatsyeva, L., and A. V. VÄhäkangas: Hardy inequalities in Triebel-Lizorkin spaces II. Aikawa dimension. - Ann. Mat. Pura Appl. (4), 2013, DOI:10.1007/s10231-013-0385-z.

[19] Käenmäki, A., J. LehrbÄCK, and M. VuOrinen: Dimensions, Whitney covers, and tubular neighborhoods. - Indiana Univ. Math. J. (to appear).

[20] Kim, P., and A. MimicA: Harnack inequalities for subordinate Brownian motions. - Electron. J. Probab. 17:37, 2012, 23.

[21] Koskela, P., and X. Zhong: Hardy's inequality and the boundary size. - Proc. Amer. Math. Soc. 131:4, 2003, 1151-1158 (electronic).

[22] Kufner, A., and L.-E. Persson: Weighted inequalities of Hardy type. - World Scientific Publishing Co. Inc., River Edge, NJ, 2003.

[23] LehrBÄCK, J.: Hardy inequalities and Assouad dimensions. - arXiv:1402.6134, 2014. 
[24] LehrbäcK, J.: Weighted Hardy inequalities beyond Lipschitz domains. - Proc. Amer. Math. Soc. 142:5, 2014, 1705-1715.

[25] Lehrbäck, J., and H. Tuominen: A note on the dimensions of Assouad and Aikawa. - J. Math. Soc. Japan 65:2, 2013, 343-356.

[26] Lewis, J. L.: Uniformly fat sets. - Trans. Amer. Math. Soc. 308:1, 1988, 177-196.

[27] Loss, M., and C. SloAne: Hardy inequalities for fractional integrals on general domains. - J. Funct. Anal. 259:6, 2010, 1369-1379.

[28] LuUkKainen, J.: Assouad dimension: antifractal metrization, porous sets, and homogeneous measures. - J. Korean Math. Soc. 35:1, 1998, 23-76.

[29] Martio, O.: Definitions for uniform domains. - Ann. Acad. Sci. Fenn. Ser. A I Math. 5:1, 1980, 197-205.

[30] Martio, O., and J. VÄısÄLÄ: Bounded turning and passability. - Results Math. 24, 1993, $246-273$.

[31] Mattila, P.: Geometry of sets and measures in Euclidean spaces. - Cambridge Stud. Adv. Math. 44, Cambridge Univ. Press, Cambridge, 1995.

[32] NeČAS, J.: Sur une méthode pour résoudre les équations aux dérivées partielles du type elliptique, voisine de la variationnelle. - Ann. Sc. Norm. Super. Pisa Cl. Sci. (3) 16, 1962, $305-326$.

[33] ŠIKić, H., R. Song, and Z. VondraČek: Potential theory of geometric stable processes. Probab. Theory Related Fields 135:4, 2006, 547-575.

[34] Slodne, C. A.: A fractional Hardy-Sobolev-Maz'ya inequality on the upper halfspace. - Proc. Amer. Math. Soc. 139:11, 2011, 4003-4016.

[35] Stein, E. M.: Singular integrals and differentiability properties of functions. - Princeton Math. Ser. 30, Princeton Univ. Press, Princeton, N.J., 1970.

[36] VÄISÄLÄ, J.: Uniform domains. - Tohoku Math. J. (2) 40:1, 1988, 101-118.

[37] Wannebo, A.: Hardy inequalities. - Proc. Amer. Math. Soc. 109:1, 1980, 85-95.

Received 22 July 2013 • Accepted 14 March 2014 